\title{
Modern Concepts in Material Science
}

\author{
Ray Hua Horng* \\ Institute of Electronics, National Chiao Tung University, Taiwan
}

*Corresponding author: Ray Hua Horng, Department of Electronics Engineering, Institute of Electronics, National Chiao Tung University, Taiwan.

Received Date: November 27, 2019

Published Date: December 04, 2019

\section{Opinion}

With the improved technologies day by day, it will influence the development and evolution of today society and make life more convenient. From the data center, Internet of Things, robots, selfdriving cars, artificial intelligence, smart medical care, Geriatric care etc., to future implementations in high-frequency communication, energy management, high-speed computing, and even innovative technologies that use more electronic information to develop various functions of serving humanity is inseparable from the application and combination of semiconductors. Therefore, the breakthrough growth of semiconductor technology will be the key to the future development of science and technology.

After many years development, semiconductor industries have accumulated countless experiences, technologies, and products. If we can grasp the growth needs of power semiconductors and combine the advantages of the integration of the industrial chain, it will be able to gain further opportunities in the future of the semiconductor technologies.

What is more worthy of attention is that in order to respond to the continued development or improvement of electronic products in the future, better semiconductor components are needed to meet their needs. Among them, the emerging materials, e.g. wide bandgap high quality single crystal like Ga203 based materials used in semiconductor components are the core elements for improving the performance of components and products.

At present, mainstream semiconductor materials are still silicon-based semiconductors based on silicon (Si). Facing the trend of circuit miniaturization, they have reached the limit in terms of process or function matching, and it is increasingly difficult to meet chip size. Diversified performance requirements such as shrinking, complex circuit functions, and high heat dissipation efficiency; coupled with more high-frequency, high-power related electronic applications in the future, and semiconductor components that require more power saving, lower operating costs, and can integrate more functionality.

Silicon-based semiconductors are limited by the physical properties of silicon and have bottlenecks that are not easy to break through. Therefore, at present, many semiconductor factories in the world are rushing to develop the materials, processes, and applications of compound semiconductors, focusing on new materials with better performance to solve the original highfrequency and high-power silicon-based semiconductors.

Potential materials for current power semiconductors, such as gallium nitride (GaN) and silicon carbide ( $\mathrm{SiC}$ ), are "wide bandgap" materials with energy band gaps greater than 3.0, which not only have better resistance to electric fields. When applied to highvoltage components, it is not easy to be crushed by an electric field, and has good electrical conductivity and heat dissipation, which can reduce energy loss, and below a low dielectric constant, it can also reduce parasitic capacitance, reduce signal delay and power consumption. Therefore, these wide bandgap semiconductor materials like Ga203 are more suitable for power devices. They take advantage of their higher efficiency, high conductivity, and heat resistance to meet the application needs of human livelihood, industry, and transportation, and overcome more stringent requirements. Use environment.

New generation oxide materials have more advantages in performance and will also affect the development of the semiconductor wafer market. It is a niche that wide bandgap semiconductor wafers have the high opportunity to go into market.

The target market for wide bandgap semiconductors with 6 to 8-inch wafers is that the current supply and manufacturing process of wide bandgap materials is only stable below 6-inch wafers (including 6-inch wafers), which is also a stage of investment by international semiconductor factories. In addition to the practical development 
and application of compound semiconductors, in addition to circuit design different from silicon-based semiconductors, more research and testing and verification are still needed. At the same time, the supply of equipment and manufacturing methods that can respond to wide bandgap materials in the process need to be strengthened. Insufficient technical staff and experience have caused problems such as low production yields. Therefore, the production cost of wide bandgap semiconductors today is quite high. Compared with the same 6-inch wafer, the initial price can be nearly 100 times higher than that of silicon wafers. Therefore, the 8-inch wide-gap semiconductor wafer that is yet to be developed is a high potential opportunity in the new technologies.

\section{Acknowledgement}

None.

\section{Conflict of Interest}

No conflict of interest. 